\title{
Sleep Disturbances and Associated Factors in Drug-Naïve Patients with Parkinson's Disease
}

\author{
Min Zhong $\mathbb{D}^{\prime}$ \\ Xu Jiang' \\ Sha Zhu' \\ Ruxin Gu' \\ $\mathrm{Yu} \mathrm{Bai}{ }^{2}$ \\ Hong $\mathrm{He}^{3}$ \\ Yang Pan' \\ Pingyi $\mathrm{Xu}^{4}$ \\ Jun Yan' \\ Li Zhang' \\ 'Department of Geriatric Neurology, \\ Affiliated Brain Hospital of Nanjing \\ Medical University, Nanjing, People's \\ Republic of China; ${ }^{2}$ Department of \\ Biological Sciences, University of Toronto \\ Scarborough, Toronto, ON, Canada; \\ ${ }^{3}$ School of Medical Instrument and Food \\ Engineering, University of Shanghai for \\ Science and Technology, Shanghai, \\ People's Republic of China; ${ }^{4}$ Department \\ of Neurology, First Affiliated Hospital of \\ Guangzhou Medical University, \\ Guangzhou, People's Republic of China
}

Correspondence: Li Zhang; Jun Yan Department of Geriatric Neurology, Affiliated Brain Hospital of Nanjing Medical University, No. 264 Guangzhou Road, Nanjing, Jiangsu Province, 210029,

People's Republic of China

Email neuro_zhangli@।63.com;

815473709@qq.com
Purpose: Sleep disturbance is one of the common symptoms in Parkinson's disease (PD). The study of sleep disturbance used to concentrate on treated PD. This study aimed to investigate the factors that are associated with the sleep quality of drug-naïve patients with PD.

Patients and Methods: All participants were interviewed using a standard questionnaire to collect basic information. PD severity, depression symptoms, anxiety symptoms, sleep quality, cognitive status, life quality, and the presence of rapid eye movement (REM) sleep behavior disorder (RBD) and minor hallucination were assessed using corresponding rating scales. The patients with a Pittsburgh Sleep Quality Index score $\leq 6$ fell into the poor sleep group, and those with REM Sleep Behavior Disorder Screening Questionnaire score $\geq 5$ were considered to have probable RBD.

Results: Seventy drug-naive patients with PD and 30 healthy controls matched for age, sex, and education were recruited. Up to $41.4 \%$ of the patients suffered from sleep disturbance, and $24.3 \%$ of the patients had RBD. Poor sleepers were more likely to have left-side predominant motor symptoms. Compared with good sleepers, poor sleepers, particularly female patients, had more burden in the aspect of anxiety and depression. RBD was associated with more nonmotor symptoms, poor sleep quality, bad performance in cognition orientation domain, anxiety, depression, presence of minor hallucination, and poor life quality.

Conclusion: Sleep disturbances are common in drug-naïve PD and require wide attention. Motor symptom laterality and gender difference in mood are associated with sleep quality. Depression, anxiety, and RBD are highly related to sleep disturbance. RBD has many comorbidities, which can influence the cognitive function and life quality of the patients.

Keywords: Parkinson's disease, drug naïve, sleep disturbance, REM sleep behavior disorder, depression, anxiety

\section{Introduction}

Parkinson's disease (PD) is the second most common neurodegenerative disorder ${ }^{1}$ and affects approximately 10 million people worldwide. Patients with PD suffer from a wide variety of nonmotor symptoms (NMS), among which sleep disorders are very common and occur in over $90 \%$ of patients with PD throughout the disease course. ${ }^{2}$ Sleep disorders in PD consist of insomnia, rapid eye movement (REM) sleep behavior disorder (RBD), excessive daytime sleepiness, restless leg syndrome, and sleep disordered breathing. Some of these sleep problems can emerge in the early stage and even the premotor stage of the disease, and become prevalent in the late stage. ${ }^{3}$

In levodopa-treated patients with $\mathrm{PD}$, sleep disruption is related with greater NMS burden and poorer life quality. ${ }^{4}$ More central PD-related pain, ${ }^{5}$ higher levodopa intake, ${ }^{2}$ longer disease duration, ${ }^{6}$ poorer cognition, ${ }^{7}$ and more fatigue ${ }^{8}$ are 
associated with worse sleep quality. Sleep disturbances and mood disorders often appear as comorbidities. ${ }^{9}$ Impairment of sleep quality and sleep continuity will make the subject prone to the development of mood disorders, and vice versa.

RBD was first formally described by Schenck et al in $1986 .{ }^{10}$ It is a sleep disorder characterized by dream enactment behavior and REM sleep without atonia. ${ }^{11}$ The violent behavior during attacks can cause harm to patients and their bedmates. ${ }^{12}$ As a special kind of sleep disorder in $\mathrm{PD}, \mathrm{RBD}$ is a strong predictor of $\alpha$-synuclein pathology. ${ }^{11}$ The prevalence of RBD in PD is between $30 \%$ and $50 \%$. In early $\mathrm{PD}$, patients with RBD have poorer subjective motor symptoms and life quality, as well as increased severity and frequency of nonmotor features, than those without RBD. ${ }^{13}$

Anti-PD medications and disease duration have considerable effect on sleep quality. In this regard, drug-naïve PD patients are an ideal cohort to evaluate the relationship between sleep disturbance and clinical characteristics, which is essential to provide evidence support for early intervention. Therefore, the present study aimed to investigate the influencing factors of sleep disorders in drugnaïve PD patients.

\section{Patients and Methods Patients and Study Design}

A cohort of newly diagnosed patients with drug-naïve PD were recruited from the neurology outpatient clinics of Nanjing Brain Hospital from April 2019 to July 2020. The patients were diagnosed by at least two experienced movement disorder specialists. Participants were eligible for inclusion if they met the following criteria: they were newly diagnosed according to the UK PD Society Brain Bank criteria $^{14}$ and did not use any anti-PD medication; they did well in the standard acute levodopa challenge test with more than $30 \%$ improvement rate in Unified Parkinson's Disease Rating Scale part III (UPDRS III) score; they had early- or middle-stage PD (Hoehn-Yahr [H-Y] stage $\leq 3$ ); and they can accomplish a series of questionnaires completely and accurately. Patients were excluded if they had any of the following: other sleep disorders that cannot be explained by PD, including sleep disorders caused by shift work, systemic diseases, or other causes; a history of major psychiatric diseases; usage of any antipsychotic medication; and abnormal vision or abnormal corrected vision. Finally, 73 patients were included, but only 70 patients accomplished the one- year follow-up. The one-year rule was strictly enforced to distinguish dementia with Lewy bodies (DLB) and PD with dementia. ${ }^{15}$ Thirty healthy controls (HC) matched for age, sex, and education were recruited from volunteers. An MRI scan was conducted on all subjects on the day of the interview to exclude the focal abnormalities, such as brain tumor and cerebrovascular diseases.

This study was approved and supervised by the local ethics committee of the Brain Hospital Affiliated to Nanjing Medical University and was conducted in accordance with the ethical standards outlined in the Helsinki Declaration. Voluntary written informed consent and clinical data were obtained from all subjects.

\section{Clinical Outcomes}

Each patient with PD was interviewed regarding age, gender, marital status, education level, body mass index (BMI), motor symptom dominant side, age at PD onset, and disease duration at baseline. The severity of motor symptoms was assessed using UPDRS III and the H-Y scale. Standard acute levodopa challenge test was performed, and UPDRS III was tested twice. The first assessment was performed after the patients fasted overnight (off state), and the second assessment was conducted $1 \mathrm{~h}$ after the patients were administered with $200 \mathrm{mg}$ levodopa (on state). ${ }^{16}$ NMS were evaluated using the Non-Motor Symptom Questionnaire (NMS-Quest). ${ }^{17}$ Global and subdomain cognitive functions were measured using Montreal Cognitive Assessment (MoCA) ${ }^{18}$ The severity of anxiety and depression were evaluated using the Hamilton Anxiety Rating Scale (HAMA) and the Hamilton Rating Scale for Depression (HAMD), respectively. Health-related quality of life was measured using the Parkinson's Disease Questionnaire-39 (PDQ39). ${ }^{19}$ The "Hallucinations and Psychosis" item of the Movement Disorder Society-sponsored revision of the Unified Parkinson's Disease Rating Scale (MDS-UPDRS) Part I was used as the screening tool to assess the presence of minor hallucination $(\mathrm{MH}) . \mathrm{MH}$ is the earliest and most frequent psychotic symptom in PD and includes presence hallucination, passage hallucination, and illusion. ${ }^{20}$ Parkinson's Disease Sleep Scale (PDSS) ${ }^{21}$ and Pittsburgh Sleep Quality Index (PSQI) ${ }^{22}$ were used to measure sleep quality, and Rapid eye movement sleep behavior disorder Screening Questionnaire (RBDSQ) was used to screen for $\mathrm{RBD}^{23}$ Patients with PSQI $\geq 6$ were identified as poor sleepers, and those with RBDSQ $\geq 5$ were considered to have probable RBD (pRBD). 
The data of the HC, including age, gender, marital status, education level, and BMI, were collected. All participants accomplished a series of assessment scales, including HAMA, HAMD, MoCA, RBDSQ, and PISQ. The presence of $\mathrm{MH}$ was also assessed.

\section{Statistical Analysis}

Continuous data are shown as mean \pm standard deviation (SD), and categorical data are shown as percentages. Shapiro-Wilk test was used to evaluate data normality. Mann-Whitney $U$-test and Chi square test were conducted to examine differences between two groups. Due to the difference of age between patients with and without RBD, scores of the assessment scales were compared after adjustment for confounding factors using the One-way Analysis of Covariance
(ANCOVA). Spearman's rank correlation coefficients were obtained to evaluate correlations. Two-sided $\mathrm{P}<0.05$ was considered statistically significant. Data analysis was performed in IBM SPSS 23.0 and GraphPad Prism version 8.0.1.

\section{Results}

\section{Clinical Variables of Participants}

We recruited 70 drug-naïve patients with PD (33 [47.1\%] males and 37 [52.9\%] females), and $30 \mathrm{HC}$ (13 [43.3\%] males and 17 [56.7\%] females). As shown in Table 1, the two groups were consistent in age, gender, percentage of living alone, educational level, and BMI. Compared with the HC group, the PD group had higher HAMA, HAMD, RBDSQ, and $\mathrm{PSQI}$ scores and $\mathrm{MH}$ prevalence $(\mathrm{P}=0.002, \mathrm{P}<0.0001$, $\mathrm{P}=0.026, \mathrm{P}=0.025, \mathrm{P}=0.009$, respectively). However, no

Table I Comparison Between PD and HC Groups

\begin{tabular}{|c|c|c|c|c|c|}
\hline & & PD & $\mathrm{HC}$ & $\chi^{2} / Z$ Value & $\mathbf{P}$ \\
\hline Number, n (\%) & & $70(70.0 \%)$ & $30(30.0 \%)$ & & \\
\hline Age, $y$, mean $\pm S D$ & & $66.76 \pm 8.47$ & $65.77 \pm 7.65$ & 938.5 & $0.40 \mathrm{I}^{\mathrm{a}}$ \\
\hline Gender, n (\%) & $\begin{array}{l}\text { Male } \\
\text { Female }\end{array}$ & $\begin{array}{l}33(47.1 \%) \\
37(52.9 \%)\end{array}$ & $\begin{array}{l}13(43.3 \%) \\
17(56.7 \%)\end{array}$ & 0.123 & $0.726^{b}$ \\
\hline Percentage of live alone, $\mathrm{n}$ (\%) & & $8(11.4 \%)$ & $2(6.7 \%)$ & 0.524 & $0.719^{b}$ \\
\hline Education, n (\%) & $\begin{array}{l}\text { Illiterate } \\
\text { Primary school } \\
\text { Middle school } \\
\text { College or above }\end{array}$ & $\begin{array}{l}15(21.4 \%) \\
9(12.9 \%) \\
35(50.0 \%) \\
11(15.7 \%)\end{array}$ & $\begin{array}{l}I(3.3 \%) \\
I 2(40.0 \%) \\
I 6(53.3 \%) \\
I(3.3 \%)\end{array}$ & 970 & $0.515^{b}$ \\
\hline $\mathrm{BMI}$, mean $\pm \mathrm{SD}$ & & $24.01 \pm 2.96$ & $24.80 \pm 2.50$ & 847 & $0.127^{\mathrm{a}}$ \\
\hline Age at onset, $y$, mean $\pm S D$ & & $64.49 \pm 8.93$ & - & & \\
\hline Disease duration, $y$, mean $\pm S D$ & & $1.71 \pm 1.43$ & - & & \\
\hline $\mathrm{H}-\mathrm{Y}$ stage, mean $\pm \mathrm{SD}$ & & $2.03 \pm 0.56$ & - & & \\
\hline UPDRS III on, mean \pm SD & & $|5.77 \pm 8.0|$ & - & & \\
\hline UPDRS III off, mean \pm SD & & $24.31 \pm 12.03$ & - & & \\
\hline $\mathrm{MH}, \mathrm{n}(\%)$ & & $18(25.7 \%)$ & $I(3.3 \%)$ & 6.835 & $0.009^{b}$ \\
\hline HAMA, mean $\pm S D$ & & $5.90 \pm 4.53$ & $2.93 \pm 2.16$ & 643.5 & $0.002^{a}$ \\
\hline HAMD, mean \pm SD & & $7.01 \pm 4.46$ & $3.23 \pm 1.59$ & 492 & $<0.0001^{a}$ \\
\hline MoCA, mean $\pm S D$ & & $24.7 I \pm 4.16$ & $24.83 \pm 3.02$ & 1000 & $0.705^{a}$ \\
\hline RBDSQ, mean \pm SD & & $2.81 \pm 2.80$ & $1.23 \pm 1.04$ & 761 & $0.026^{a}$ \\
\hline PSQI, mean \pm SD & & $5.93 \pm 4.61$ & $3.40 \pm 2.46$ & 752.5 & $0.025^{a}$ \\
\hline
\end{tabular}

Notes: ${ }^{a}$ mann-Whitney U-test. ${ }^{b}$ Chi-square test. Significant results are highlighted in bold $(\mathrm{p}<0.05)$.

Abbreviations: PD, Parkinson's disease; HC, healthy control; SD, standard deviation; BMI, body mass index; H-Y stage, Hoehn and Yahr stage; UPDRS III, the Unified Parkinson's Disease Rating Scale part III; MH, minor hallucination; HAMA, Hamilton Anxiety Rating Scale; HAMD, Hamilton Depression Rating Scale; MoCA, Montreal Cognitive Assessment; RBDSQ, the Rapid eye movement sleep behavior disorder Screening Questionnaire; PSQI, Pittsburgh Sleep Quality Index. 
significant difference was observed in the MoCA scores of the groups ( $\mathrm{P}=0.705)$; the mean MoCA scores of the $\mathrm{PD}$ and $\mathrm{HC}$ groups were $24.71 \pm 4.16$ and $24.83 \pm 3.02$, respectively.

\section{Differences Between Good and Poor}

\section{Sleepers}

Patients were divided into two groups using a cut-off value of 6 points in PSQI. The good sleepers had higher PDSS scores, as well as lower HAMA and HAMD scores than the poor sleepers ( $\mathrm{P}=0.005,0.006,0.007$, respectively). The two groups had differences in the laterality of motor symptoms $(\mathrm{P}=0.017)$. Most of the poor sleepers had more severe leftside motor symptoms, whereas the good sleepers had rightside dominance. The results are shown in Table 2. Gender, percentage of living alone, educational level, BMI, age at PD onset, and disease duration were similar between the two groups. Motor symptoms, (ie, H-Y stage and UPDRS III on and off scores), NMS (ie, NMS-Quest, MoCA, and RBDSQ

Table 2 Comparisons of Clinical Characteristics and Demographics According to PSQI Scores

\begin{tabular}{|c|c|c|c|c|c|}
\hline & & PSQI < 6 & PSQI $\geq 6$ & $\chi^{2} / Z$ Value & $\mathbf{P}$ \\
\hline Number, n (\%) & & $4 \mathrm{I}(58.6 \%)$ & $29(41.4 \%)$ & & \\
\hline Age, $y$, mean $\pm S D$ & & $66.07 \pm 8.00$ & $67.72 \pm 9.15$ & 510.5 & $0.316^{\mathrm{a}}$ \\
\hline Gender, n (\%) & $\begin{array}{l}\text { Male } \\
\text { Female }\end{array}$ & $\begin{array}{l}20(48.8 \%) \\
2 I(5 I .2 \%)\end{array}$ & $\begin{array}{l}13(44.8 \%) \\
16(55.2 \%)\end{array}$ & 0.107 & $0.744^{b}$ \\
\hline Percentage of live alone, $\mathrm{n}(\%)$ & & $3(7.3 \%)$ & $5(17.2 \%)$ & 1.629 & $0.202^{b}$ \\
\hline Education, n (\%) & $\begin{array}{l}\text { Illiterate } \\
\text { Primary school } \\
\text { Middle school } \\
\text { College or above }\end{array}$ & $\begin{array}{c}7(17.1 \%) \\
5(12.2 \%) \\
20(48.8 \%) \\
9(22.0 \%)\end{array}$ & $\begin{array}{c}8(27.6 \%) \\
4(13.8 \%) \\
15(51.7 \%) \\
2(6.9 \%)\end{array}$ & 469 & $0.107^{b}$ \\
\hline $\mathrm{BMI}$, mean $\pm \mathrm{SD}$ & & $24.32 \pm 2.73$ & $23.57 \pm 3.26$ & 508.5 & $0.305^{\mathrm{a}}$ \\
\hline Motor symptom dominant side $\mathrm{n}(\%)$ & $\begin{array}{l}\text { None } \\
\text { Left } \\
\text { Right }\end{array}$ & $\begin{array}{c}3(7.3 \%) \\
16(39.0 \%) \\
22(53.7 \%)\end{array}$ & $\begin{array}{c}0(0.0 \%) \\
19(65.5 \%) \\
10(34.5 \%)\end{array}$ & 5.732 & $0.017^{b}$ \\
\hline Age at onset, $y$, mean $\pm S D$ & & $63.98 \pm 8.16$ & $65.21 \pm 10.03$ & 535.5 & $0.481^{a}$ \\
\hline Disease duration, $y$, mean $\pm S D$ & & $1.62 \pm 1.09$ & $1.86 \pm 1.82$ & 589 & $0.947^{\mathrm{a}}$ \\
\hline $\mathrm{H}-\mathrm{Y}$ stage, mean $\pm \mathrm{SD}$ & & $2.00 \pm 0.60$ & $2.07 \pm 0.5 ।$ & 563 & $0.688^{a}$ \\
\hline UPDRS III on, mean \pm SD & & $15.20 \pm 8.42$ & $16.59 \pm 7.47$ & 525.5 & $0.410^{a}$ \\
\hline UPDRS III off, mean \pm SD & & $23.49 \pm 12.23$ & $25.48 \pm 11.85$ & 529 & $0.434^{\mathrm{a}}$ \\
\hline NMS-Quest, mean \pm SD & & $9.98 \pm 5.64$ & $12.24 \pm 5.32$ & 471.5 & $0.142^{a}$ \\
\hline PDSS, mean \pm SD & & $\mid 18.40 \pm 31.23$ & $109.76 \pm 16.80$ & 358 & $0.005^{a}$ \\
\hline MoCA, mean $\pm S D$ & & $24.98 \pm 4.15$ & $24.34 \pm 4.21$ & 536.5 & $0.486^{a}$ \\
\hline HAMA, mean $\pm S D$ & & $4.68 \pm 3.91$ & $7.62 \pm 4.85$ & 364 & $0.006^{a}$ \\
\hline HAMD, mean \pm SD & & $5.80 \pm 3.98$ & $8.72 \pm 4.61$ & 369 & $0.007^{\mathrm{a}}$ \\
\hline $\mathrm{PDQ} 39$, mean $\pm \mathrm{SD}$ & & $32.61 \pm 24.87$ & $34.03 \pm 19.79$ & 544.5 & $0.551^{a}$ \\
\hline RBDSQ, mean \pm SD & & $2.93 \pm 2.92$ & $2.66 \pm 2.68$ & 574 & $0.804^{\mathrm{a}}$ \\
\hline $\mathrm{MH}, \mathrm{n}(\%)$ & & $9(22.0 \%)$ & $9(31.0 \%)$ & 0.734 & $0.392^{b}$ \\
\hline
\end{tabular}

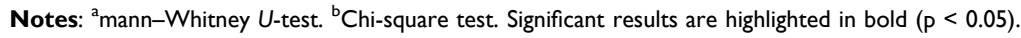

Abbreviations: PSQI, Pittsburgh Sleep Quality Index; SD, standard deviation; BMI, body mass index; H-Y stage, Hoehn and Yahr stage; UPDRS III, the Unified Parkinson's Disease Rating Scale part III; NMS-Quest, Non-Motor Symptoms Questionnaire; PDSS, The Parkinson's Disease Sleep Scale; MoCA, Montreal Cognitive Assessment; HAMA, Hamilton Anxiety Rating Scale; HAMD, Hamilton Depression Rating Scale; PDQ39, Parkinson's Disease Questionnaire-39; RBDSQ, the Rapid eye movement sleep behavior disorder Screening Questionnaire; MH, minor hallucination. 
scores), MH prevalence, and life quality were also not remarkably different between the two groups.

\section{Gender Difference in Anxiety and Depression Symptoms}

We compared the gender differences of patients with emotional symptoms. HAMA and HAMD scores were significantly higher in female patients with sleep problem than those without sleep problem $(\mathrm{P}=0.007,0.035$, respectively). Emotional differences between male and female patients with or without sleep problem were not obvious (Table 3).

\section{Differences Between Patients with and without RBD}

Approximately $24.3 \%$ of the patients with drug-naïve PD had pRBD. The demographic data of patients with and without pRBD were consistent, except for age. The patients without pRBD were slightly older than the patients with $\mathrm{pRBD}(\mathrm{P}=0.042)$. When we performed the comparison of NMS and life quality between groups, age was adjusted using one-way ANCOVA. The NMS-Quest and PDSS scores were calculated excluding the sleep domain and the nocturnal psychosis domain scores, respectively. The patients with pRBD suffered from NMS $(P=0.001)$ than those without, especially in PDSS, HAMA, and HAMD scores and MH prevalence $(\mathrm{P}=0.028,0.001,0.003,0.027$, respectively). Global cognition was not different between groups $(\mathrm{P}=0.125)$, whereas the pRBD group performed worse than the non$\mathrm{RBD}$ group in orientation domain $(\mathrm{P}=0.028)$. The results are shown in Table 4.

\section{Correlations Between Sleep Disorders and NMS}

In Figure 1, PSQI score in the PD group was associated with $\operatorname{HAMA}\left(\mathrm{r}_{\mathrm{s}}=0.358, \mathrm{P}=0.002\right), \operatorname{HAMD}\left(\mathrm{r}_{\mathrm{s}}=0.379, \mathrm{P}=0.001\right)$, and PDSS scores $\left(\mathrm{r}_{\mathrm{s}}=-0.534, \mathrm{P}<0.0001\right)$. RBDSQ score was correlated with HAMA $\left(r_{s}=0.247, P=0.039\right), \operatorname{HAMD}\left(r_{s}=\right.$ $0.274, \mathrm{P}=0.021)$, NMS-Quest $\left(\mathrm{r}_{\mathrm{s}}=0.328, \mathrm{P}=0.006\right)$, PDQ39 score $\left(r_{s}=0.360, P=0.002\right)$, and MOCA-orientation domain score $\left(r_{\mathrm{s}}=-0.276, \mathrm{P}=0.021\right)$. RBDSQ score was not associated with PDSS scores $\left(r_{s}=-0.233, P=0.052\right)$.

\section{Discussion}

Sleep disturbances, emotional disorders, and MHs were highly more prevalent in PD than in healthy age-matched control population, even before medical treatment was initiated. These symptoms may be related to each other and belong to a cluster in PD.

The prevalence of sleep disturbances in PD ranges between $20 \%$ and $98 \% .^{24,25}$ The wide range is due to differences in populations and assessment scales. In a sample of 491 treated patients with PD, $29.9 \%$ of them suffered from sleep disturbance as PDSS was used to quantify sleep quality. ${ }^{25}$ Fernandes et al studied 1589 patients with PD from different centers using an NMS scale and found that $45.3 \%$ had sleep problems. ${ }^{26}$ Melka et al surveyed 155 treated patients with $\mathrm{PD}$, all of whom reported having sleep problems from time to time, and $43.9 \%$ had a PDSS2 score $>18 .{ }^{24}$ In the present study, $41.4 \%$ of the drug-naïve patients had poor sleep quality based on PSQI score. Multiple factors contribute to sleep disorders in PD. The dopamine dysfunction of the hypothalamus, a key area in the regulation of the sleep-wake cycle, may be one of the factors. Altered peripheral clock gene expression in earlystage PD may contribute to the change of serum hormone level, such as melatonin and cortisol, which consequently lead to sleep rhythm disturbance and sleep efficiency reduction in patients. ${ }^{27}$ Dopaminergic treatment can partially improve sleep disorders by increasing sleeping time and improving nocturnal akinesia. ${ }^{28}$ However, this relief is not complete, and the prevalence of sleep disturbance still increases as the disease progresses. ${ }^{29}$

Table 3 Comparisons of HAMA and HAMD Scores Between Good and Poor Sleeper Based on Gender

\begin{tabular}{|c|c|c|c|c|c|c|c|c|c|}
\hline \multicolumn{2}{|c|}{ Variable } & \multirow[t]{2}{*}{ Non-SP } & \multirow[t]{2}{*}{ SP } & \multicolumn{2}{|c|}{ Non-SP vs SP } & \multicolumn{2}{|c|}{ Non-SP(Male) vs Non-SP(Female) } & \multicolumn{2}{|c|}{ SP(Male) vs SP(Female) } \\
\hline & & & & Z Value & $\mathbf{P}$ & Z Value & $\mathbf{P}$ & Z Value & $\mathbf{P}$ \\
\hline HAMA & $\begin{array}{l}\text { Male } \\
\text { Female }\end{array}$ & $\begin{array}{l}4.50 \pm 4.09 \\
4.86 \pm 3.84\end{array}$ & $\begin{array}{l}5.69 \pm 3.97 \\
9.19 \pm 5.05\end{array}$ & $\begin{array}{l}100.5 \\
81\end{array}$ & $\begin{array}{l}0.28 \mathrm{I}^{\mathrm{a}} \\
0.007^{\mathrm{a}}\end{array}$ & 198 & $0.751^{a}$ & 63 & $0.075^{a}$ \\
\hline HAMD & $\begin{array}{l}\text { Male } \\
\text { Female }\end{array}$ & $\begin{array}{l}5.30 \pm 4.21 \\
6.29 \pm 3.80\end{array}$ & $\begin{array}{l}7.23 \pm 3.61 \\
9.94 \pm 5.08\end{array}$ & $\begin{array}{l}91 \\
99\end{array}$ & $\begin{array}{l}0.158^{a} \\
0.035^{a}\end{array}$ & 170.5 & $0.300^{\mathrm{a}}$ & 80.5 & $0.308^{a}$ \\
\hline
\end{tabular}

Notes: ${ }^{a}$ mann-Whitney $U$-test. Significant results are highlighted in bold $(p<0.05)$.

Abbreviations: HAMA, Hamilton Anxiety Rating Scale; HAMD, Hamilton Depression Rating Scale; SP, sleep problem. 
Table 4 Demographic Information and Clinical Characters of the Patients with and without RBD

\begin{tabular}{|c|c|c|c|c|c|}
\hline & & PRBD & non-pRBD & $\chi^{2} /$ Z/F Value & $\mathbf{P}$ \\
\hline \multicolumn{2}{|l|}{ Number, n (\%) } & $17(24.3 \%)$ & $53(75.7 \%)$ & & \\
\hline \multicolumn{2}{|l|}{ Age, $y$, mean $\pm S D$} & $63.00 \pm 9.51$ & $67.96 \pm 7.83$ & 302 & $0.042^{a}$ \\
\hline Gender, n (\%) & $\begin{array}{l}\text { Male } \\
\text { Female }\end{array}$ & $\begin{array}{c}10(58.8 \%) \\
7(41.2 \%)\end{array}$ & $\begin{array}{l}23(43.4 \%) \\
30(56.6 \%)\end{array}$ & 1.229 & $0.268^{b}$ \\
\hline \multicolumn{2}{|l|}{ Percentage of live alone, n (\%) } & I(5.9\%) & $7(13.2 \%)$ & 0.673 & $0.669^{b}$ \\
\hline Education, n (\%) & $\begin{array}{l}\text { Illiterate } \\
\text { Primary school } \\
\text { Middle school } \\
\text { College or above }\end{array}$ & $\begin{array}{l}6(35.3 \%) \\
1(5.9 \%) \\
8(47.1 \%) \\
2(11.8 \%)\end{array}$ & $\begin{array}{c}9(17.0 \%) \\
8(15.1 \%) \\
27(50.9 \%) \\
9(17.0 \%)\end{array}$ & 381 & $0.304^{b}$ \\
\hline \multicolumn{2}{|l|}{ BMI, mean \pm SD } & $23.22 \pm 2.60$ & $24.26 \pm 3.05$ & 342.5 & $0.139^{a}$ \\
\hline Motor symptom dominant side, $\mathrm{n}(\%)$ & $\begin{array}{l}\text { None } \\
\text { Left } \\
\text { Right }\end{array}$ & $\begin{array}{c}0(0.0 \%) \\
8(47.1 \%) \\
9(52.9 \%)\end{array}$ & $\begin{array}{c}3(5.7 \%) \\
27(50.9 \%) \\
23(43.4 \%)\end{array}$ & 0.012 & $0.913^{b}$ \\
\hline \multicolumn{2}{|l|}{ Age of onset, $y$, mean $\pm S D$} & $60.88 \pm 9.71$ & $65.64 \pm 8.44$ & 308.5 & $0.052^{a}$ \\
\hline \multicolumn{2}{|l|}{ Disease duration, $y$, mean $\pm S D$} & $1.87 \pm 1.26$ & $1.67 \pm 1.49$ & 388 & $0.383^{\mathrm{a}}$ \\
\hline \multicolumn{2}{|l|}{$\mathrm{H}-\mathrm{Y}$ stage, mean $\pm \mathrm{SD}$} & $1.97 \pm 0.4 \mathrm{I}$ & $2.05 \pm 0.61$ & 399 & $0.45 \mathrm{I}^{\mathrm{a}}$ \\
\hline \multicolumn{2}{|l|}{ UPDRS III on, mean \pm SD } & $17.18 \pm 6.96$ & $15.32 \pm 8.33$ & 358.5 & $0.207^{\mathrm{a}}$ \\
\hline \multicolumn{2}{|l|}{ UPDRS III off, mean \pm SD } & $23.75 \pm 12.85$ & $26.06 \pm 9.14$ & 371.5 & $0.279^{a}$ \\
\hline \multicolumn{2}{|l|}{ NMS-Quest ${ }^{d}$, mean \pm SD } & $\mid 1.71 \pm 3.85$ & $7.92 \pm 4.74$ & 13.093 & $0.00 I^{c}$ \\
\hline \multicolumn{2}{|l|}{$\mathrm{PDSS}^{\mathrm{e}}$, mean $\pm \mathrm{SD}$} & $93.71 \pm 21.96$ & $103.00 \pm 17.50$ & 5.070 & $0.028^{c}$ \\
\hline \multicolumn{2}{|l|}{ MoCA, mean $\pm S D$} & $24.29 \pm 4.06$ & $24.85 \pm 4.22$ & 2.412 & $0.125^{c}$ \\
\hline & $\begin{array}{l}\text { Visuospatial/executive } \\
\text { Naming } \\
\text { Attention } \\
\text { Language } \\
\text { Abstraction } \\
\text { Delayed memory } \\
\text { Orientation }\end{array}$ & $\begin{array}{l}3.47 \pm I .33 \\
2.88 \pm 0.33 \\
5.4 I \pm 0.94 \\
2.7 I \pm 0.77 \\
I .35 \pm 0.6 I \\
3.4 I \pm I .37 \\
5.06 \pm I .25\end{array}$ & $\begin{array}{l}3.28 \pm 1.57 \\
2.81 \pm 0.44 \\
5.51 \pm 0.85 \\
2.81 \pm 0.52 \\
1.53 \pm 0.70 \\
3.25 \pm 1.19 \\
5.66 \pm 0.88\end{array}$ & $\begin{array}{l}0.041 \\
<0.0001 \\
1.440 \\
1.567 \\
1.940 \\
0.451 \\
5.063\end{array}$ & $\begin{array}{l}0.840^{c} \\
0.993^{c} \\
0.234^{c} \\
0.215^{c} \\
0.168^{c} \\
0.504^{c} \\
0.028^{c}\end{array}$ \\
\hline \multicolumn{2}{|l|}{ HAMA, mean \pm SD } & $7.94 \pm 5.57$ & $5.52 \pm 3.99$ & 11.166 & $0.001^{c}$ \\
\hline \multicolumn{2}{|l|}{ HAMD, mean \pm SD } & $9.12 \pm 4.66$ & $6.34 \pm 4.22$ & 9.293 & $0.003^{c}$ \\
\hline \multicolumn{2}{|l|}{ PDQ39, mean \pm SD } & $45.18 \pm 19.37$ & $29.36 \pm 22.58$ & 12.191 & $0.001^{c}$ \\
\hline \multicolumn{2}{|l|}{ PSQI, mean \pm SD } & $6.18 \pm 5.59$ & $5.85 \pm 4.31$ & 0.212 & $0.646^{c}$ \\
\hline \multicolumn{2}{|l|}{ MH, n (\%) } & $8(47.1 \%)$ & $10(18.9 \%)$ & 5.131 & $0.027^{c}$ \\
\hline
\end{tabular}

Notes: ${ }^{a}$ mann-Whitney U-test. ${ }^{b}$ Chi-square test. ${ }^{c}$ The value and $\mathrm{P}$ are calculated adjusted for age. ${ }^{\mathrm{d}}$ The scores were calculated from the NMS-Quest total score excluding the sleep domain score. ${ }^{e}$ The scores were calculated from the PDSS total score excluding the nocturnal psychosis domain score. Significant results are highlighted in bold $(\mathrm{p}<0.05)$. Abbreviations: RBD, Rapid eye movement sleep disorder; SD, standard deviation; BMI, body mass index; H-Y stage, Hoehn and Yahr stage; UPDRS III, the Unified Parkinson's Disease Rating Scale part III; NMS-Quest, Non-Motor Symptoms Questionnaire; PDSS, The Parkinson's Disease Sleep Scale; MoCA, Montreal Cognitive Assessment; HAMA, Hamilton Anxiety Rating Scale; HAMD, Hamilton Depression Rating Scale; PDQ39, Parkinson's Disease Questionnaire-39; PSQI, Pittsburgh Sleep Quality Index; MH, minor hallucination.

Previous studies found that patients with left-dominant motor symptom were more likely to be poor sleepers. ${ }^{31,32}$ Our previous finding using wearable device in objective gait assessment also confirmed this finding. ${ }^{30}$ In the present study, we found poor sleepers tend to be left-dominant in motor symptom which proves from another perspective 

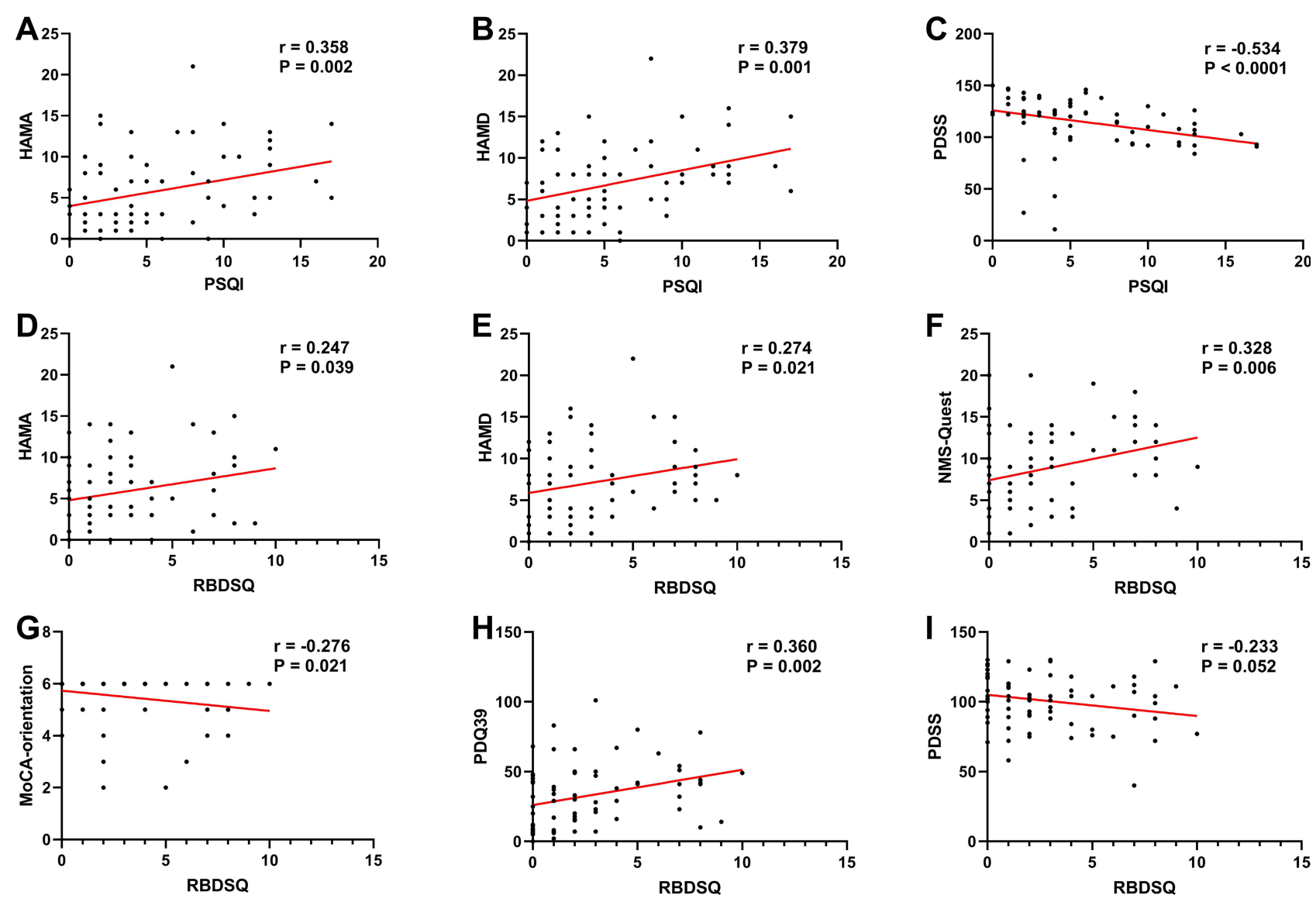

Figure I Correlations among PSQI/RBDSQ scores and nonmotor symptoms in patients with PD.

Notes: A significant correlation between (A) PSQI and HAMA in total patients $\left(r_{s}=0.358, P=0.002\right) ;(B)$ PSQI and HAMD in total patients $\left(r_{s}=0.379, P=0.001\right) ;(C)$ PSQI and PDSS in total patients $\left(r_{s}=-0.534, P<0.0001\right)$; (D) RBDSQ and HAMA in total patients $\left(r_{s}=0.247, P=0.039\right) ;(E)$ RBDSQ and HAMD in total patients $\left(r_{s}=\right.$ $0.274, P=0.021)$; (F) RBDSQ and NMS-Quest in total patients $\left(r_{s}=0.328, P=0.006\right)$; the NMS-Quest scores were calculated excluding the sleep domain scores; $(\mathbf{G})$ RBDSQ and MoCA-orientation in total patients $\left(r_{s}=-0.276, P=0.021\right)$; $(\mathbf{H})$ RBDSQ and PDQ39 in total patients $\left(r_{s}=0.360, P=0.002\right)$; $(\mathbf{I})$ RBDSQ and PDSS in total patients $\left(r_{s}=-0.233, P=0.052\right)$; the PDSS scores were calculated excluding the nocturnal psychosis domain score.

Abbreviations: PSQI, Pittsburgh Sleep Quality Index; RBDSQ, the Rapid eye movement sleep behavior disorder Screening Questionnaire; PD, Parkinson's disease; HAMA, Hamilton Anxiety Rating Scale; HAMD, Hamilton Depression Rating Scale; PDSS, The Parkinson's Disease Sleep Scale; NMS-Quest, Non-Motor Symptoms Questionnaire; MOCA-orientation, Montreal Cognitive Assessment-orientation domain; PDQ39, Parkinson's Disease Questionnaire-39; $r_{s}$, Spearman's rank correlation coefficient.

that the lateralization of motor symptoms and sleep quality are corelated in patients with PD. Under normal circumstances, the right hemisphere is more responsible for the dopamine secretion than the left hemisphere and contains a neural network related to vigilance and awakening. The uneven dopamine secretion and uneven distribution of sleep-related neural circuits maybe a possible explanation for the relationship between left-dominant motor symptoms and sleep disorders.

The relationship between sleep disorders and mood disorders has been widely confirmed in different populations beyond PD patients, ${ }^{4-6,25,33}$ and these comorbidities have mutual influence. ${ }^{9}$ In drug-naïve patients with $\mathrm{PD}$, poor sleepers were more likely to experience anxiety and depression, and we found this phenomenon is more substantial in female patients, which was consistent with
Mahale's finding. ${ }^{34}$ These results may suggest that sleep disorders and mood disorders partially share pathophysiological mechanisms. The damage and pathological changes of neurotransmitters, such as serotonin, norepinephrine, and dopamine, in brainstem nuclei may result in this relationship. ${ }^{25}$

As the strongest prodromal marker of $\alpha$-synucleinopathy, ${ }^{35}$ RBD has attracted much attention. It is associated with higher risks of dementia, autonomic failure, severe motor symptom, and life quality burden. ${ }^{36,37}$ In our study, RBD had a prevalence of $24.3 \%$ in drug-naïve PD patients, and is related with decreased sleep quality, anxiety, depression, impaired cognition in orientation domain, reduced life quality, and severe NMS, especially autonomic failure. The results were similar with an observational study conducted by Liu et al. ${ }^{38}$ The mechanism of RBD is not very clear. Postmortem and 
neuroimaging studies in patients with RBD revealed that the key structures for REM sleep are located in the mesopontine tegmentum and the ventral medial medulla (VMM). ${ }^{39,40}$ Other structures, such as the thalamus, basal forebrain, limbic areas, and frontal cortex, are also involved. ${ }^{41}$ Numerous animal experiments and human research reported that RBD occurs because of damage in the nucleus, such as the glutamatergic subcoeruleus/sublateral dorsal nucleus, and/or GABAergic and glycinergic VMM, or lesions in connections between the two. $^{41,42}$ Impairment of the connections of the subcoeruleus nucleus and VMM with other structures may also explain the pathophysiology of RBD. ${ }^{41}$

The relationship between RBD symptoms and other clinical characters in patients with $\mathrm{PD}$ remains controversial. Iranzo et $\mathrm{al}^{41}$ proposed from a different perspective that the relationship of RBD with comorbid depression and autonomic failure cannot indicate a causal relationship; these factors are just compatible in timing sequence. This may be true because according to the hypothesis of Braak, $^{43} \alpha$-synuclein initially accumulates in the dorsal motor nucleus of the glossopharyngeal and vagal nerves and anterior olfactory nucleus, subsequently affects the medulla and pons, and finally spreads to the cortex, which eventually causes the comorbidity of RBD with other symptoms. Evidence from multimodality imaging 44 and mouse model of $\mathrm{RBD}^{45}$ proved this hypothesis.

In the present study, we improved the knowledge on sleep disorders in patients with drug-naïve PD. This study has several limitations. First, the number of patients was small, and a referral bias might have influenced the results. Larger samples are needed to confirm our results. Second, this work is a cross-sectional study. Sleep disturbances are a common symptom in PD. Therefore, a longitudinal study is beneficial to understand the fluctuations in sleep quality and the effects on disease progression. Third, as the gold standard for the diagnosis of sleep disorders, polysomnography was not performed in all patients and was not included in this study.

\section{Conclusion}

The sleep quality of patients with $\mathrm{PD}$ requires more attention. Our results confirmed the high prevalence of sleep disturbances, especially RBD, in patients with drug-naïve PD. Based on our previous research, we further confirmed the effect of motor symptom laterality on sleep disturbances in drug-naive patients. Sleep quality, cognition orientation domain, mood, and life quality are affected in patients with RBD. Therefore, identifying RBD in clinics is very important because it has many comorbidities and has great effects on patients.

\section{Data Sharing Statement}

The data supporting the findings of this study are included in the article, further inquiries can be directed to the corresponding authors.

\section{Statement of Ethics}

The study was approved by the Ethics committee of Nanjing Brain Hospital Affiliated to Nanjing Medical University. All patients signed a written informed consent before the study. All procedures followed the declaration of Helsinki.

\section{Acknowledgments}

We thank the patients and their families for their participation in the study.

\section{Author Contributions}

All authors made a significant contribution to the work reported, whether that is in the conception, study design, execution, acquisition of data, analysis and interpretation, or in all these areas; took part in drafting, revising or critically reviewing the article; gave final approval of the version to be published; have agreed on the journal to which the article has been submitted; and agree to be accountable for all aspects of the work.

\section{Funding}

This work was supported by the Special Funds of the Jiangsu Provincial Cadre Health Projects [grant number BJ20005, BJ17006], the Jiangsu Provincial Key Research and Development Program [grant numbers BE2018610, BE2019612], the Nanjing Medical Science and Technology Development Foundation [grant number QRX17026] and the Nanjing Rehabilitation Medicine Center Project [grant number is not available].

\section{Disclosure}

The authors declare that they have no conflict of interest.

\section{References}

1. Connolly BS, Lang AE. Pharmacological treatment of Parkinson disease: a review. JAMA. 2014;311(16):1670-1683. doi:10.1001/ jama.2014.3654

2. Schaeffer E, Vaterrodt T, Zaunbrecher L, et al. Effects of Levodopa on quality of sleep and nocturnal movements in Parkinson's Disease. J Neurol. 2021;268(7):2506-2514. doi:10.1007/s00415-021-10419-7 
3. Suzuki K, Okuma Y, Uchiyama T, et al. Impact of sleep-related symptoms on clinical motor subtypes and disability in Parkinson's disease: a multicentre cross-sectional study. J Neurol Neurosurg Psychiatry. 2017;88(11):953-959. doi:10.1136/jnnp-2017-316136

4. Santos-Garcia D, Castro ES, de Deus Fonticoba T, et al. Sleep Problems Are Related to a Worse Quality of Life and a Greater NonMotor Symptoms Burden in Parkinson's Disease. J Geriatr Psychiatry Neurol. 2021;34(6):642-658. doi:10.1177/0891988720964250.

5. Vila-Cha N, Cavaco S, Mendes A, et al. Sleep disturbances in Parkinson's disease are associated with central parkinsonian pain. J Pain Res. 2019;12:2137-2144. doi:10.2147/JPR.S206182

6. Chang CW, Fan JY, Chang BL, Wu YR. Anxiety and Levodopa Equivalent Daily Dose Are Potential Predictors of Sleep Quality in Patients With Parkinson Disease in Taiwan. Front Neurol. 2019;10:340. doi:10.3389/fneur.2019.00340

7. Neikrug AB, Maglione JE, Liu L, et al. Effects of sleep disorders on the non-motor symptoms of Parkinson disease. J Clin Sleep Med. 2013;9(11):1119-1129. doi:10.5664/jcsm.3148

8. Siciliano M, Trojano L, De Micco R, et al. Motor, behavioural, and cognitive correlates of fatigue in early, de novo Parkinson disease patients. Parkinsonism Relat Disord. 2017;45:63-68. doi:10.1016/j. parkreldis.2017.10.004

9. Miniati M, Palagini L, Maglio A, Marazziti D, Dell'Osso L. Can sleep disturbance be a cue of mood spectrum comorbidity? A preliminary study in panic disorder. CNS Spectr. 2020;25 (1):32-37. doi:10.1017/S1092852918001700

10. Schenck C, Bundlie S, Ettinger M, Mahowald M. Chronic behavioral disorders of human REM sleep: a new category of parasomnia. Sleep. 1986;9(2):293-308. doi:10.1093/sleep/9.2.293

11. Antelmi E, Lippolis M, Biscarini F, Tinazzi M, Plazzi G. REM sleep behavior disorder: mimics and variants. Sleep Med Rev. 2021;60:101515. doi:10.1016/j.smrv.2021.101515

12. Howell MJ, Schenck CH. Rapid Eye Movement Sleep Behavior Disorder and Neurodegenerative Disease. JAMA Neurol. 2015;72 (6):707-712. doi:10.1001/jamaneurol.2014.4563

13. Rolinski M, Szewczyk-Krolikowski K, Tomlinson PR, et al. REM sleep behaviour disorder is associated with worse quality of life and other nonmotor features in early Parkinson's disease. J Neurol Neurosurg Psychiatry. 2014;85(5):560-566. doi:10.1136/jnnp-2013-306104

14. Hughes A, Daniel S, Kilford L, Lees A. Accuracy of clinical diagnosis of idiopathic Parkinson's disease: a clinico-pathological study of 100 cases. J Neurol Neurosurg Psychiatry. 1992;55(3):181-184. doi:10.1136/jnnp.55.3.181

15. Arnaoutoglou NA, O'Brien JT, Underwood BR. Dementia with Lewy bodies - from scientific knowledge to clinical insights. Nat Rev Neurol. 2019;15(2):103-112. doi:10.1038/s41582-018-0107-7

16. Wu Z, Jiang X, Zhong M, et al. Wearable Sensors Measure Ankle Joint Changes of Patients with Parkinson's Disease before and after Acute Levodopa Challenge. Parkinsons Dis. 2020;2020:2976535. doi:10.1155/2020/2976535

17. Chaudhuri KR, Martinez-Martin P, Schapira AH, et al. International multicenter pilot study of the first comprehensive self-completed nonmotor symptoms questionnaire for Parkinson's disease: the NMSQuest study. Mov Disord. 2006;21(7):916-923. doi:10.1002/ mds. 20844

18. Gill DJ, Freshman A, Blender JA, Ravina B. The Montreal cognitive assessment as a screening tool for cognitive impairment in Parkinson's disease. Mov Disord. 2008;23(7):1043-1046. doi:10.1002/mds.22017

19. Peto V, Jenkinson C, Fitzpatrick R, Greenhall R. The development and validation of a short measure of functioning and well being for individuals with Parkinson's disease. Qual Life Res. 1995;4 (3):241-248. doi:10.1007/BF02260863

20. Lenka A, Pagonabarraga J, Pal PK, Bejr-Kasem H, Kulisvesky J. Minor hallucinations in Parkinson disease: a subtle symptom with major clinical implications. Neurology. 2019;93(6):259-266. doi:10.1212/WNL.0000000000007913
21. Chaudhuri K, Pal S, DiMarco A, et al. The Parkinson's disease sleep scale: a new instrument for assessing sleep and nocturnal disability in Parkinson's disease. J Neurol Neurosurg Psychiatry. 2002;73 (6):629-635. doi:10.1136/jnnp.73.6.629

22. Buysse D, Reynolds C, Monk T, Berman S, Kupfer D. The Pittsburgh Sleep Quality Index: a new instrument for psychiatric practice and research. Psychiatry Res. 1989;28(2):193-213. doi:10.1016/01651781(89)90047-4

23. Stiasny-Kolster K, Mayer G. The REM sleep behavior disorder screening questionnaire-a new diagnostic instrument. Mov Disord. 2007;22(16):2386-2393. doi:10.1002/mds.21740

24. Melka D, Tafesse A, Bower JH, Assefa D. Prevalence of sleep disorders in Parkinson's disease patients in two neurology referral hospitals in Ethiopia. BMC Neurol. 2019;19(1):205. doi:10.1186/ s12883-019-1431-2

25. Zhu J, Zhong M, Yan J, et al. Depressive symptoms effect subjective sleep quality in Chinese patients with Parkinson's disease. Clin Neurol Neurosurg. 2020;195:105950. doi:10.1016/j. clineuro.2020.105950

26. Fernandes M, Pierantozzi M, Stefani A, et al. Frequency of Nonmotor Symptoms in Parkinson's Patients With Motor Fluctuations. Front Neurol. 2021;12:678373. doi:10.3389/fneur.2021.678373

27. Breen DP, Vuono R, Nawarathna U, et al. Sleep and circadian rhythm regulation in early Parkinson disease. JAMA Neurol. 2014;71 (5):589-595. doi:10.1001/jamaneurol.2014.65

28. Seppi K, Weintraub D, Coelho M, et al. The Movement Disorder Society Evidence-Based Medicine Review Update: treatments for the non-motor symptoms of Parkinson's disease. Mov Disord. 2011;26 (Suppl 3):S42-80. doi:10.1002/mds.23884

29. Ou R, Hou Y, Wei Q, et al. Longitudinal evolution of non-motor symptoms in early Parkinson's disease: a 3-year prospective cohort study. NPJ Parkinsons Dis. 2021;7(1):58. doi:10.1038/s41531-02100207-5

30. Zhu S, Zhong M, Bai Y, et al. The Association Between Clinical Characteristics and Motor Symptom Laterality in Patients With Parkinson's Disease. Front Neurol. 2021;12:663232. doi:10.3389/ fneur.2021.663232

31. Cubo E, Martinez-Martin P, Gonzalez-Bernal J, et al. Effects of Motor Symptom Laterality on Clinical Manifestations and Quality of Life in Parkinson's Disease. J Parkinsons Dis. 2020;10 (4):1611-1620. doi:10.3233/JPD-202067

32. Stavitsky K, McNamara P, Durso R, Harris E, Auerbach S, CroninGolomb A. Hallucinations, dreaming, and frequent dozing in Parkinson disease: impact of right-hemisphere neural networks. Cogn Behav Neurol. 2008;21(3):143-149. doi:10.1097/ WNN.0b013e318185e698

33. Nyer M, Farabaugh A, Fehling K, et al. Relationship between sleep disturbance and depression, anxiety, and functioning in college students. Depress Anxiety. 2013;30(9):873-880. doi:10.1002/ da. 22064

34. Mahale R, Yadav R, Pal PK. Does gender differences have a role in determining sleep quality in Parkinson's disease? Acta Neurol Belg. 2020;121(4):1001-1007. doi:10.1007/s13760-020-01498-w

35. Dauvilliers Y, Schenck CH, Postuma RB, et al. REM sleep behaviour disorder. Nat Rev Dis Primers. 2018;4(1):19. doi:10.1038/s41572018-0016-5

36. Schrempf W, Brandt MD, Storch A, Reichmann H. Sleep disorders in Parkinson's disease. J Parkinsons Dis. 2014;4(2):211-221. doi:10.3233/JPD-130301

37. Zhu J, Lu L, Zhong M, et al. Increased rapid eye movement density in Chinese patients with Parkinson's disease and RBD. Neurol Sci. 2021;42(3):961-968. doi:10.1007/s10072-020-04597-x

38. Liu H, Ou R, Wei Q, et al. Rapid eye movement behavior disorder in drug-naive patients with Parkinson's disease. J Clin Neurosci. 2019;59:254-258. doi:10.1016/j.jocn.2018.07.007 
39. Ehrminger M, Latimier A, Pyatigorskaya N, et al. The coeruleus/ subcoeruleus complex in idiopathic rapid eye movement sleep behaviour disorder. Brain. 2016;139(Pt 4):1180-1188. doi:10.1093/brain/ aww006

40. Iranzo A, Tolosa E, Gelpi E, et al. Neurodegenerative disease status and post-mortem pathology in idiopathic rapid-eye-movement sleep behaviour disorder: an observational cohort study. Lancet Neurol. 2013;12(5):443-453. doi:10.1016/S1474-4422(13)70056-5

41. Iranzo A. The REM sleep circuit and how its impairment leads to REM sleep behavior disorder. Cell Tissue Res. 2018;373(1):245-266. doi: 10.1007/s00441-018-2852-8

42. St Louis EK, Boeve BF, Sleep Behavior REM. Disorder: diagnosis, Clinical Implications, and Future Directions. Mayo Clin Proc. 2017;92(11):1723-1736. doi:10.1016/j.mayocp.2017.09.007
43. Braak H, Del Tredici K, Rüb U, de Vos R, Jansen Steur E, Braak E. Staging of brain pathology related to sporadic Parkinson's disease. Neurobiol Aging. 2003;24(2):197-211. doi:10.1016/S0197-4580(02) 00065-9

44. Knudsen K, Fedorova TD, Hansen AK, et al. In-vivo staging of pathology in REM sleep behaviour disorder: a multimodality imaging case-control study. Lancet Neurol. 2018;17(7):618-628. doi:10.1016/ S1474-4422(18)30162-5

45. Shen Y, Yu WB, Shen B, et al. Propagated alpha-synucleinopathy recapitulates REM sleep behaviour disorder followed by parkinsonian phenotypes in mice. Brain. 2020;143(11):3374-3392. doi:10.1093/brain/awaa283

\section{Publish your work in this journal}

Neuropsychiatric Disease and Treatment is an international, peerreviewed journal of clinical therapeutics and pharmacology focusing on concise rapid reporting of clinical or pre-clinical studies on a range of neuropsychiatric and neurological disorders. This journal is indexed on PubMed Central, the 'PsycINFO' database and CAS, and is the official journal of The International Neuropsychiatric Association (INA). The manuscript management system is completely online and includes a very quick and fair peer-review system, which is all easy to use. Visit http://www.dovepress.com/testimonials.php to read real quotes from published authors. 